\title{
Desarrollo y aplicación de una metodología para la elaboración de mapas de peligro municipales por deslizamientos de terreno mediante Sistemas de Información Geográfica
}

\author{
Edgar M. Uribe ${ }^{\mathrm{a}^{*}}$, Francisco Corral ${ }^{\mathrm{b}, 1,2}$, José C. Escamilla $^{\mathrm{a}}$, Enrique Cruz ${ }^{\mathrm{a}}$ \\ ${ }^{a}$ Área Académica de Ciencias de la Tierra y Materiales, Universidad Autónoma del Estado de Hidalgo, Carr. Pachuca-Tulancingo km 4.5, Col. Carboneras, Mineral \\ de la Reforma, Hidalgo, C.P. 42184 \\ ${ }^{\mathrm{b}}$ Estudiante de Licenciatura en Ingeniería en Geología Ambiental, Área Académica de Ciencias de la Tierra y Materiales, Universidad Autónoma del Estado de \\ Hidalgo, Carr. Pachuca-Tulancingo km 4.5, Col. Carboneras, Mineral de la Reforma, Hidalgo, C.P. 42184
}

\section{Resumen}

Se propone y aplica una metodología para la identificación de zonas propensas a deslizamientos mediante el uso de Sistemas de Información Geográfica (SIG). El Centro Nacional de Prevención de Desastres (CENAPRED) de México propone una metodología que debe aplicarse ladera por ladera, que consiste en asignar un puntaje que refleje la susceptibilidad de cada uno de los factores relevantes para los procesos de deslizamientos, como pendiente, uso de suelo, tipo de roca, etc. La mayor desventaja de esta metodología es que resulta en un análisis discreto (un puntaje por ladera), cuando actualmente contamos con SIG que pueden ser usados para asignar puntajes en regiones completas que resultan naturalmente en mapas de índices de peligro. Además los SIG permiten realizar esta labor de forma expedita, eficiente, y sin necesidad de realizar visitas de campo, aunque aún se sugiere realizarlas para afinar información, calibrar la metodología y validar los reusltados. En el presente artículo exploramos la aplicación de la metodología propuesta por CENAPRED pero mediante SIG. Debido a que hay algunos factores que no pueden ser calculados en SIG, como la altura de un talud (no confundir con altimetría), los factores aquí empleados difieren de los factores propuestos por CENAPRED. Los factores aquí empleados son los siguientes: pendiente, tipo de roca, tipo de suelo, fallas, uso de suelo, y ocurrencia previa de deslizamientos. Todos ellos se obtuvieron de fuentes de información públicas oficiales. La metodología se aplicó en Angangueo, Michoacán, y permitió crear un mapa de peligro para toda el área de interés. Dicho mapa captura adecuadamente las zonas donde han ocurrido deslizamientos con mayor frecuencia. Estas zonas corresponden a la zona urbana, donde además la topografía es muy accidentada. Y, por otra parte, también se logra identificar correctamente las zonas con menor riesgo, que están asociadas a tierras de cultivo con pendientes suaves, donde no se han presentado deslizamientos. Por lo cual, se considera que la metodología propuesta permite potenciar la metodología propuesta por CENAPRED para permitir la elaboración de mapas de peligro. Quizás el mayor reto para su aplicación a escala municipal, es la disponibilidad de cartografía de alta resolución. Además, el uso de suelo ofrece también un gran reto por su gran dinamismo, asociado a la deforestación y urbanización. Por lo cual, se considera importante que las instituciones encargadas de la cartografía nacional oficial actualicen la información con la mayor frecuencia posible.

Palabras Clave: Mapas de Riesgo Municipales por deslizamiento, Mapas de Peligro Municipales por deslizamiento, Deslizamientos, Sistemas de Información Geográfica, Angangueo, Michoacán, Inestatabilidad de Laderas, Procesos de Remoción de Masa.

\section{Introducción}

El peligro de deslizamientos es de suma relevancia, dada su alta frecuencia. A diferencia de sismos catastróficos, que ocurren con poca frecuencia, los deslizamientos ocurren varias veces al año. Además, se espera que su ocurrencia continúe incrementando pues los factores que los provocan están aumentando en frecuencia e intensidad. Por ejemplo, las zonas urbanas crecen descontroladamente, y la población cada vez habita lugares más peligrosos, dada su falta de regulación y la facilidad para ser invadidos. Además, se espera que las lluvias torrenciales, que son un factor detonador de deslizamientos, aumenten en intensidad y frecuencia por causa del Cambio Climático Global (IPCC, 2014).

Una de las herramientas indispensables para mitigar el peligro por deslizamiento es el uso de mapas de peligro. Se espera que estos mapas nos permitan identificar zonas de alto riesgo para evitar su urbanización o para evacuarlas, en caso de que ya estén habitadas. En México, el El Centro Nacional de Prevención de Desastres (CENAPRED) es la institución responsable de proponer metodologías para la elaboración de mapas de riesgo (CENAPRED, 2004). Desafortunadamente, algunas de estas metodologías son

\footnotetext{
* Autor en correspondencia.

Correo electrónico: edgar_uribe8920@uaeh.edu.mx
} 
obsoletas, ineficientes o imposibles de aplicar (porque no se cuenta con la información necesaria). En el caso de heladas, por ejemplo, CENAPRED recomienda realizar una interpolación de temperaturas congelantes registradas en estaciones meteorológicas. Sin embargo, hay municipios que cuentan solamente con una estación meteorológica. Así que, una interpolación no es factible. En el caso de deslizamientos, por ejemplo, CENAPRED plantea aplicar una metodología de puntuación de factores ladera por ladera, lo cual puede resultar ineficiente en términos de tiempo, especialmente en la actualidad, cuando contamos con Sistemas de Información Geográfica (SIG). Estos nos permiten realizar análisis similares sin necesidad de salir a campo, y no sólo para laderas individuales sino para municipios o áreas de estudio completas. Es el caso de mapas de peligro por inundación y otros fenómenos, que han sido desarrollados mediante SIG (Uribe, 2010).

Este artículo propone el desarrollo y aplicación de una metodología basada en SIG para la elaboración de mapas de peligro por deslizamientos. Se ha decidido que la zona de estudio sea el municipio de Angangueo, Michoacán. Angangueo es un municipio que ha sufrido múltiples deslizamientos catastróficos que han resultado en significativas pérdidas humanas y económicas (Hernández, 2014), debido a que el asentamiento urbano se encuentra situado en una zona con relieve accidentado.

\section{Zona de Estudio}

La zona de estudio corresponde a Mineral de Angangueo, Michoacán (Figura 1), que se encuentra entre los paralelos $19^{\circ} 35^{\prime}$ y $19^{\circ} 41^{\prime}$ de latitud norte; y los meridianos $100^{\circ} 14^{\prime}$ y $100^{\circ} 22^{\prime}$ de longitud oeste, y tiene altitudes entre 2300 y 3600 metros. El municipio de Angangueo colinda al norte con el municipio de Senguio, al este con el estado de México, al sur con el municipio de Ocampo, y al oeste con los municipios de Ocampo y Aporo. La zona se encuentra situada en la provincia fisiográfica del eje neovolcánico, con un tipo de clima templado subhúmedo con lluvias en verano, semifrío subhúmedo con lluvias en verano, y templado subhúmedo con lluvias en verano, con un rango de precipitación de 800 a $1200 \mathrm{~mm}$. El área de estudio se localiza en la parte norte del Sector Central de la Franja Volcánica Transmexicana, muy cercana al borde con el Cinturón de Pliegues y Fallas de la Sierra Madre Oriental, en el estado de Hidalgo (INEGI, 2009).

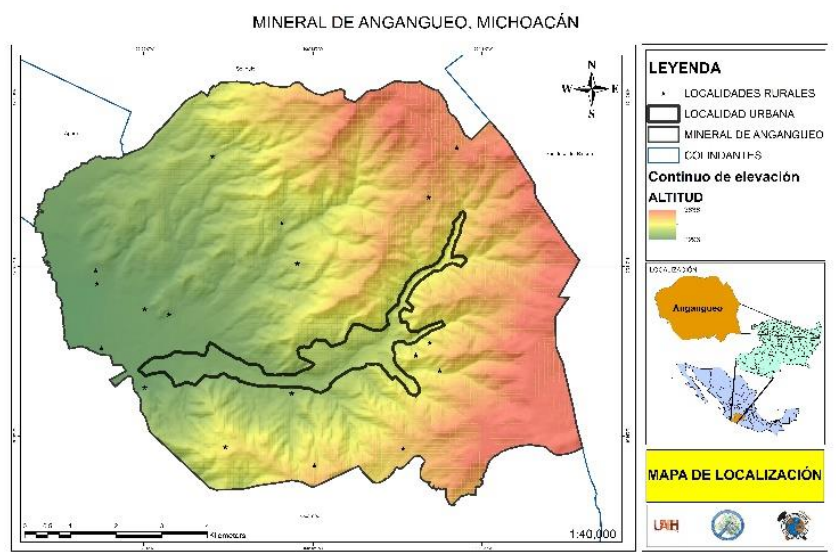

Figura 1: Ubicación del Municipio de Angangueo, Michoacán, localidades rurales (puntos negros), zona urbana (área amarilla) y Modelo de Elevación Digital (escala de colores verde a rojo).

\section{Materiales y métodos}

La metodología está parcialmente basada en la metodología propuesta por CENAPRED para la elaboración de atlas de riesgo municipales (CENAPRED, 2004). Sin embargo, a diferencia de CENAPRED, que sugiere la aplicación de la metodología a partir de visitas de campo puntuales a laderas por separado, se propone el uso de Sistemas de Información Geográfica, que permitan aplicar la metodología a toda el área de interés, haciendo el proceso más expedito y eficiente.

CENAPRED plantea la aplicación de una metodología de puntaje, es decir, identifica cada uno de los factores de peligro que son relevantes para el fenómeno de deslizamientos, y asigna una puntuación a cada factor. La puntuación de cada factor es proporcional a la susceptibilidad de generar un deslizamiento. Por ejemplo, CENAPRED propone que el valor de la puntuación de peligro y la pendiente sean proporcionales, como indica la Tabla 1. En este caso, la puntuación de peligro es mínima (0.5) para pendientes menores a $15^{\circ}$; y es máxima (2.0) para pendientes mayores a $45^{\circ}$.

CENAPRED propone aplicar ese mismo procedimiento a otros factores de peligro asociados a deslizamientos: elevación del talud, antecedentes de deslizamientos, tipo de suelos o rocas, aspectos estructurales de formaciones rocosas, evidencias geomorfológicas de huecos en laderas contiguas, vegetación y uso de la tierra, y régimen de agua en la ladera. Al igual que con la pendiente, se asigna una puntuación que sea proporcional a la factibilidad de que un factor resulte en deslizamientos. Por ejemplo, en el caso de uso de suelo, la puntuación debería ser menor para bosques que para zonas urbanas porque las primeras implican una mayor permeabilidad, que resultará en menor escurrimiento $\mathrm{y}$, en consecuencia, en menor susceptibilidad a deslizamientos. Una vez que se cuenta con las puntuaciones de cada factor, se suman, y se realiza una clasificación de esa puntuación total, donde se espera que los valores altos correspondan a una mayor susceptibilidad de deslizamientos, y viceversa.

En la medida de lo posible, se trató de usar la misma puntuación de factores de peligro propuesta por CENAPRED. Por ejemplo, en el caso de la pendiente, una vez que se calculó el mapa de pendiente, se procedió a realizar la re-clasificación propuesta por CENAPRED verbatim pues no existía ningún impedimento para ello. Sin embargo, hubo factores que no se encontraban disponibles en archivo vectorial. Así que, hubo que eliminarlos pero se incluyeron algunos otros. Es el caso de altura de talud, que frecuentemente es confundido con altimetría pero son cosas diferentes (i.e. un talud puede encontrarse a 3,000 metros sobre el nivel del mar (altimetría) pero tener solamente $300 \mathrm{~m}$ de altura). Desafortunadamente, no se conoce algún método en SIG que nos permitiera calcular la altura de los taludes de forma continua en un dominio de estudio. Así que, este factor se eliminó del análisis. Sin embargo, consideramos que la pendiente es una buena aproximación de este factor. Los factores que sí se contemplaron son los siguientes: pendiente, tipo de roca, tipo de suelo, fallas, uso de suelo, y ocurrencia previa de deslizamientos. Su fuente y puntuaciones de peligro son descritas a continuación. 
La pendiente en grados fue estimada a partir del Continuo de Elevación Mexicano 3.0 (CEM, (INEGI, 2017)). El CEM tiene un tamaño de pixel de $15 \mathrm{~m}$. A este factor se le asignaron los atributos según la Tabla 1. Como se mencionó anteriormente, esta clasificación fue adoptaba verbatim de CENAPRED.

Tabla 1: Puntuación de peligro en función de la pendiente

\begin{tabular}{lc}
\hline $\begin{array}{c}\text { Intervalo de } \\
\text { la pendiente }\end{array}$ & $\begin{array}{c}\text { Puntuación } \\
\text { de peligro }\end{array}$ \\
\hline Más de $45^{\circ}$ & 2.0 \\
$35^{\circ}$ a $45^{\circ}$ & 1.8 \\
$25^{\circ}$ a $35^{\circ}$ & 1.4 \\
$15^{\circ}$ a $25^{\circ}$ & 1.0 \\
Menos de $15^{\circ}$ & 0.5 \\
\hline
\end{tabular}

Por otro lado, para generar el factor de tipo de rocas se realizó una digitalización de los datos de Carta Geológica Minera con nombre y clave "MORELIA E14-1" del municipio de Mineral de Angangueo (Servicio Geológico Mexicano, 1998). En este caso, también se adoptó el puntaje de peligro propuesto por CENAPRED que resultó en la Tabla 2. A diferencia de la pendiente, en este caso se debe considerar un factor adicional por meteorización, además del tipo de roca. De manera que, la puntuación de peligro final resulta de la multiplicación del peligro inicial por el factor adicional por meteorización.

Tabla 2: Puntuación de peligro en función del tipo de roca

\begin{tabular}{lcl}
\hline Tipo de Roca & $\begin{array}{c}\text { Puntuación } \\
\text { inicial } \\
\text { de peligro }\end{array}$ & $\begin{array}{c}\text { Factor adicional por } \\
\text { meteorización }\end{array}$ \\
\hline $\begin{array}{l}\text { Sedimentarias } \\
\text { y tobas }\end{array}$ & 0.3 & $\begin{array}{l}\text { Multiplicar por } 1.2 \mathrm{a} \\
1.5, \text { según el grado de } \\
\text { meteorización }\end{array}$ \\
Metamórficas & 0.4 & $\begin{array}{l}\text { Multiplicar por 2 a 4, } \\
\text { Ígún el grado de } \\
\text { Igneas }\end{array}$ \\
& 0.6 & \\
\hline
\end{tabular}

Para la puntuación del factor de tipo de suelo, los datos fueron obtenidos de la Comisión Nacional para el Conocimiento y Uso de la Biodiversidad (2018) ya que estos datos contienen tipo de suelo, textura, fase física y fase química. La Tabla 3 muestra la puntuación asignada, también basada parcialmente en lo propuesto por CENAPRED.

Tabla 3: Puntuación de peligro en función del tipo de suelo

\begin{tabular}{lc}
\hline \multicolumn{1}{c}{ Tipo de Suelo } & $\begin{array}{c}\text { Puntuación } \\
\text { de peligro }\end{array}$ \\
\hline $\begin{array}{l}\text { Suelos granulares medianamente } \\
\text { compactos a sueltos. Suelos que } \\
\text { reblandecen con la adsorción de agua. }\end{array}$ & 1.5 a 2.5 \\
$\begin{array}{l}\text { Formaciones poco consolidadas. } \\
\text { Suelo arcillosos consistentes o areno } \\
\text { limosos compactos. }\end{array}$ & 0.5 a 1.0 \\
\hline
\end{tabular}

De igual forma para el factor de aspectos estructurales en formaciones rocosas se realizó una digitalización de las fallas y fracturas que presenta el territorio de Mineral de Angangueo según la carta de Angangueo, clave E14-A26 (Servicio Geológico Mexicano, 1998). A cada falla se le asignó un radio de influencia de 15 metros, y a las áreas resultantes se les asignó una puntuación de peligro igual a uno.

Para el factor de uso de suelo y vegetación se tomaron los datos de las cartas topográficas con claves E14A26 y E14A25 (INEGI, 2015). Debido a que estas cartas tenían algunas zonas sin clasificación, dichas zonas se clasificaron mediante el prontuario de información geográfica municipal de los Estados Unidos Mexicanos de Angangueo, Michoacán de Ocampo con clave geoestadística 16005 (INEGI, 2009). Desafortunadamente, el mapa resultante mostraba una zona urbana con una extensión menor a lo observado actualmente. Así que, finalmente se realizó una actualización de la zona urbana con ayuda de imágenes satelitales de Google Earth, con fecha de diciembre de 2016. La puntuación de peligro adoptada se indica en la Tabla 4.

Tabla 4: Puntuación de peligro en función del uso de suelo

\begin{tabular}{lc}
\hline \multicolumn{1}{c}{ Uso de suelo } & $\begin{array}{c}\text { Puntuación } \\
\text { de peligro }\end{array}$ \\
\hline Zona urbana & 2.0 \\
Cultivos anuales & 1.5 \\
Vegetación intensa & 0.0 \\
Rocas con raíces de arbustos en sus & 2.0 \\
fracturas & \\
Vegetación moderada & 0.8 \\
Área deforestada & 2.0 \\
\hline
\end{tabular}

Por último, en relación al factor de antecedentes de deslizamientos en el área de estudio, se sabe que la zona urbana del municipio tiene antecedentes de eventos. Por ejemplo, el día 4 de febrero del año 2010, hubo 34 personas fallecidas y 500 casas destruidas (Hernández, 2014). Por lo cual, a la zona urbana, se le asignaron puntajes de peligro con base en la Tabla 5 .

Tabla 5: Puntuación de peligro en función de la antecedencia de eventos

\begin{tabular}{lc}
\hline \multicolumn{1}{c}{$\begin{array}{c}\text { Antecedentes de } \\
\text { deslizamientos en el Área }\end{array}$} & $\begin{array}{c}\text { Puntuación } \\
\text { de peligro }\end{array}$ \\
\hline No se sabe & 0.3 \\
Algunos someros & 0.4 \\
Sí, incluso con fechas & 0.6 \\
\hline
\end{tabular}

\section{Resultados}

Para todos y cada uno de los factores se estimó la puntuación de peligro correspondiente dentro de un SIG. Por cuestión de espacio, sólo mostramos la cartografía de los puntajes de pendiente y de uso de suelo pues son los más determinantes en el mapa final de peligro (ver Figura 4). La Figura 2 muestra la puntuación de peligro para la pendiente según la clasificación discutida en la metodología. El terreno en general es bastante accidentado, a excepción de una planicie en la zona este. La pendiente en general muestra una variabilidad espacial relativamente fuerte. 


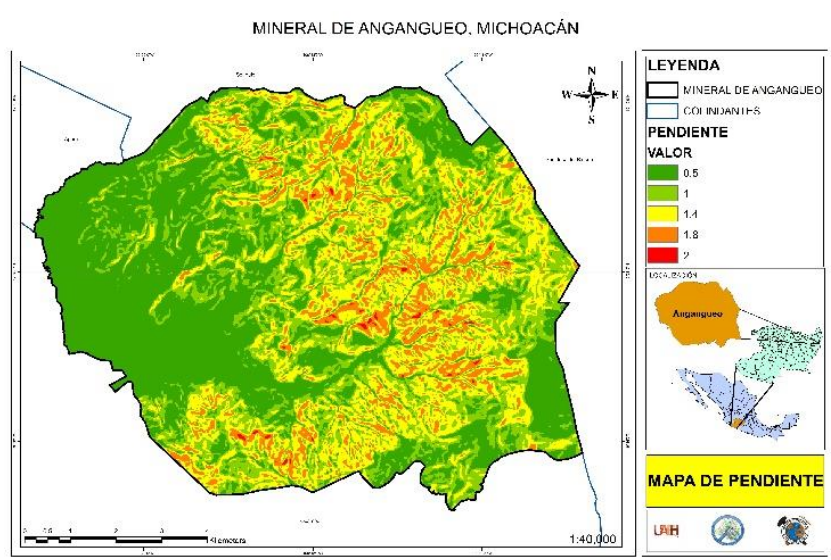

Figura 2: Mapa de puntuación de peligro para el factor de pendiente topográfica. Las zonas en rojo indican mayor susceptibilidad a deslizamientos; y las zonas verdes, lo opuesto.

La Figura 3 muestra la puntuación de peligro para el factor de uso de suelo. En este caso, hay tres categorías predominantes. La zona de menor puntuación corresponde a bosques; la zona intermedia corresponde a cultivos; y la zona de mayor puntuación corresponde a la zona urbana de Angangueo. En este caso, la variabilidad espacial es mucho más suave que la de la pendiente.

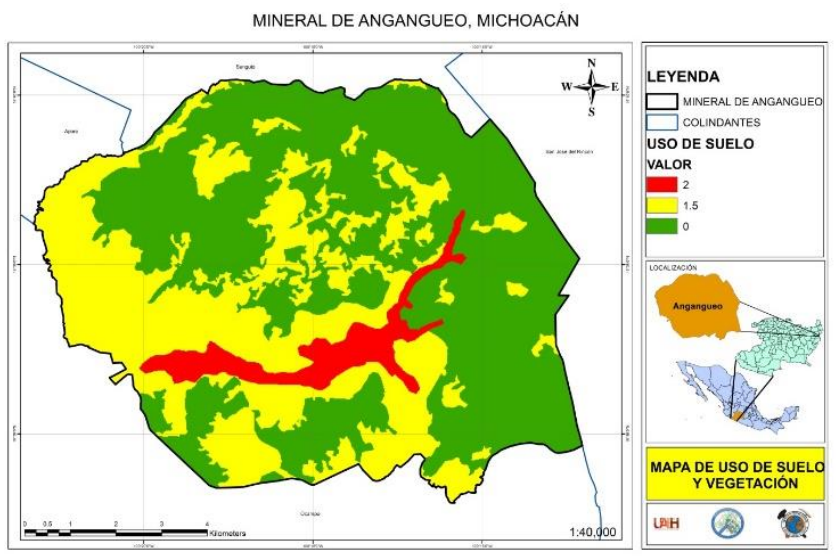

Figura 3: Mapa de puntuación de peligro para uso de suelo. Las zonas en rojo indican mayor susceptibilidad a deslizamientos; y las zonas verdes, lo opuesto.

Una vez que se contaba con todas las cartografías de las puntuaciones de peligro, se procedió a sumarlas para obtener el mapa de peligro por deslizamiento (Figura 4). A diferencia de CENAPRED, que propone crear categorías en función de intervalos regulares, lo que se propuso es usar el método de interrupciones naturales (i.e. natural breaks), que define categorías a partir de discontinuidades en el histograma de frecuencias. Estas discontinuidades son identificadas mediante desviaciones estándar en los datos. Así, se crearon seis categorías. Las zonas rojas corresponden a las zonas de mayor peligro; y las verdes, a las zonas de menor peligro. Es posible observar que los patrones resultantes están principalmente determinados por la pendiente y el uso de suelo. La zona que resulta con mayor peligro es la zona urbana, y la zona de menor peligro corresponde a la planicie en el oeste pues las pendientes son muy bajas y no existe urbanización. El mapa refleja efectivamente lo que ha ocurrido históricamente en Angangueo, y también lo que hemos observado durante los recorridos de campo.

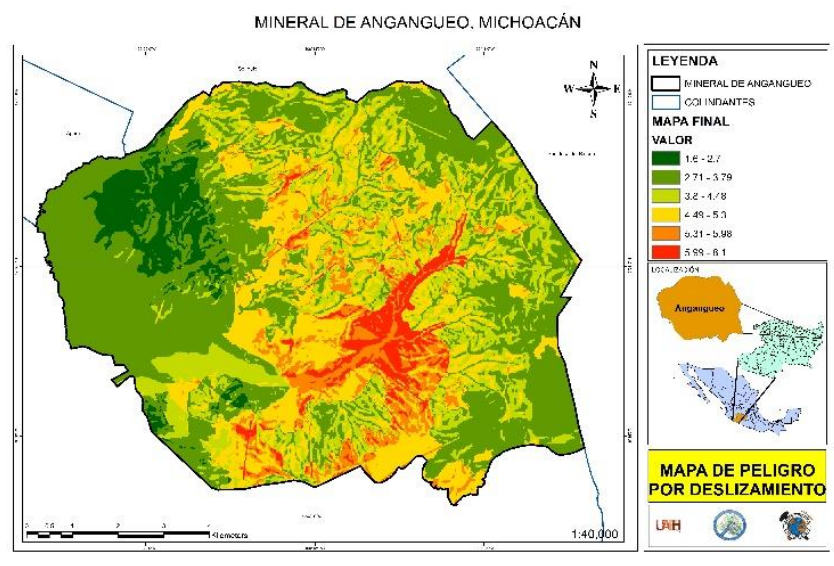

Figura 4: Mapa de peligro por deslizamiento obtenido mediante la suma de todos las puntuaciones de peligro, y clasificado en interrupciones naturales (i.e. natural breaks). Las zonas en rojo indican mayor susceptibilidad a deslizamientos; y las zonas verdes, lo opuesto.

\section{Discusión}

Se ha presentado la propuesta y aplicación de una metodología para la elaboración de mapas de peligro por deslizamientos con base en SIG en Angangueo, Michoacán. Se ha adoptado en la medida de lo posible la metodología de CENAPRED. La metodología consiste en asignar puntuaciones de peligro a cada uno de los factores asociados a deslizamientos. Sin embargo, debido a que dicha metodología se aplica a laderas individuales, hemos tenido que hacer algunas adecuaciones para poder aplicarla en SIG. Por ejemplo, no se han incluido todos los factores que propone CENAPRED porque no se cuenta con dicha información en SIG. Afortunadamente, algunos factores podrían resultar hasta cierto punto redundantes como pendiente, que sí fue incluida, y elevación del talud, que no fue incluida por no haber un método para estimarla en SIG (no confundir con altimetría). Los factores incluidos fueron: pendiente, tipo de roca, tipo de suelo, fallas, uso de suelo, y ocurrencia previa de deslizamientos.

Se considera que la metodología ha arrojado resultados satisfactorios pues refleja aquellos lugares donde históricamente se han presentado deslizamientos, y también refleja lo que han observado los autores en campo. Los factores más determinantes en el mapa de peligro final son la pendiente y el uso de suelo. La zona que se encuentra en mayor riesgo es la zona urbana pues está constituida por zonas impermeables, que generan más escurrimiento, que a su vez pueden potenciar la ocurrencia de deslizamientos. Además, la zona urbana se encuentra en un terreno muy accidentado, lo cual también propicia la inestabilidad del terreno, y resulta en mayores deslizamientos.

El uso de SIG permite la realización de mapas de peligro preliminares de forma más expedita, simple y económica, en contraste con metodologías basadas en trabajo de campo, ya que las visitas a campo implican gastos, y tiempo, tanto para traslado, como para reconocimiento. Tal es el caso de la metodología propuesta por CENAPRED, que considera la 
visita de cada ladera para el cálculo de la puntuación de peligro. Con lo anterior, no estamos sugiriendo que el trabajo de campo deba ser suprimido. Por el contrario, consideramos que el trabajo de campo es indispensable para afinar la información, así como para calibrar la metodología y validar sus resultados. Por ejemplo, algunos grados de intemperismo y meteorización no podrían ser reconocidos correctamente mediante imágenes satelitales. Por lo cual, su correcta evaluación requiere visitas de campo. Sin embargo, dentro de las ventajas de la presente metodología está la posibilidad de ofrecer un resultado preliminar de forma relativamente eficiente en términos de tiempo y costos. Y el resultado puede posteriormente emplearse como guía para identificar aquellos lugares que es indispensable visitar para afinar los resultados. Una ventaja adicional es que es posible obtener un mapa de peligro de forma natural, a diferencia de la metodología de CENAPRED, donde sólo tendríamos información de aquellas laderas que hayan sido visitadas en campo, pero no así de la totalidad del área de estudio.

Uno de los retos que enfrentamos durante la elaboración de este mapa es conseguir la información más precisa y actualizada del uso de suelo, pues la deforestación y urbanización son fenómenos sumamente dinámicos. Afortunadamente, fue posible conseguir información actualizada gracias a las imágenes de Google Earth. Podemos concluir entonces que la calidad del mapa resultante de esta metodología dependerá muchísimo de la calidad de la información integrada en el análisis. Por lo cual, es deseable que las instituciones responsables de cartografía en México, como INEGI, actualicen su información con la mayor frecuencia posible, para poder así elaborar mapas de riesgo y otros productos relacionados actualizados, que reflejen efectivamente las condiciones del área de interés.

\section{English Summary}

Development and application of a methodology for the elaboration of landslide municipal hazards maps using Geographic Information Systems

\footnotetext{
Abstract

A methodology is proposed and applied to identify areas prone to landslides through the use of Geographic Information Systems (GIS). CENAPRED proposes a methodology that has to be applied slope by slope, which consists of assigning a score that reflects the susceptibility of each of the relevant factors for landslide processes, such as slope, land use, type of rock, etc. The main disadvantage of this methodology is that it results in a discrete analysis (a score per slope), when we currently have GIS that can be used to assign scores in complete regions that naturally result in hazard index maps. In addition, GIS allows this work to be carried out expeditiously, efficiently, and without the need for field visits, although visits are still suggested to refine the input data, calibrate the methodology, and validate the results. In the present article we explore the application of the methodology proposed by CENAPRED but using SIG. Since there are some factors that cannot be
}

calculated in GIS, such as the height of a slope (not to be confused with altimetry), the factors used here differ from the factors proposed by CENAPRED. The factors used here are the following: slope, type of rock, type of soil, faults, land use, and previous occurrence of landslides. All of them were obtained from official public information sources. The methodology was applied in Angangueo, Michoacán, and allowed creating a hazard map for the entire area of interest. This map adequately captures the areas where landslides have occurred most frequently. These zones correspond to the urban area, where the topography is also very rugged. And, on the other hand, it is also possible to correctly identify areas with lower risk, which are associated with cultivated lands with gentle slopes, where no landslides have occurred. Therefore, it is considered that the proposed methodology enhance the methodology proposed by CENAPRED to allow the elaboration of continuous hazard maps. Perhaps the greatest challenge for its application at municipal level is the availability of high resolution cartography. In addition, land use cartography also offers a great challenge due to its great dynamism, associated with deforestation and urbanization. Therefore, it is considered important that the institutions in charge of official national cartography update the information as frequently as possible.

Keywords:

Landslide Municipal Risk Maps, Landslide Municipal Hazard Maps, Geographic Information Systems, Angangueo, Michoacán, Landslides, Slope Instability.

\section{Agradecimientos}

Este trabajo ha sido realizado durante el desarrollo de los proyectos de investigación: "Caracterización en un Espacio Bidimensional de Fallas y Fracturas en la Sierra de Pachuca" y del proyecto "Análisis de la lluvia como detonador de la ocurrencia de deslizamientos de terreno". Así mismo, agradecemos las valiosas contribuciones de los editores.

\section{Referencias}

CENAPRED, 2004, Guía básica para la elaboración de atlas estatales y municipales de peligro y riesgo, México, DF, 389pp.

Hernández, J. C., 2014, A un 50\% la reconstrucción de Angangueo a 4 años de la tragedia. Recuperado el 17 de 10 de 2018 , de QUADRATIN: https://www.quadratin.com.mx/principal/A-un-50-lareconstruccion-de-Angangueo-4-anos-de-la-tragedia/

INEGI, 2009, Prontuario de información geográfica municipal de los Estados Unidos Mexicanos. Recuperado el 29 de 10 de 2018, de http://www3.inegi.org.mx/contenidos/app/mexicocifras/datos_geografi cos/16/16005.pdf

INEGI, 2015, Conjunto de datos vectoriales de información topográfica escala 1:50 000 serie III. E14A26 (Angangueo). Recuperado el 29 de 10 de 2018 , de http://www.beta.inegi.org.mx/app/biblioteca/ficha.html?upc $=7028252$ 68657

INEGI, 2017, Continuo de Elevaciones Mexicano 3.0 (CEM 3.0), Recuperado de: http://www.inegi.org.mx/geo/contenidos/datosrelieve/continental/conti nuoelevaciones.aspx.

IPCC, 2014, Climate Change 2014: Synthesis Report, Geneva, Switzerland, 151pp. 
Servicio Geológico Mexicano, 1998. Carta Geólogico Minero Angangueo E14-26. Recuperado el 28 de 10 de 2018, de

https://mapserver.sgm.gob.mx/Cartas_Online/geologia/1566_E14A26 GM.pdf

Servicio Geológico Mexicano, 1998. Carta Geólogico Minera, Morelia E14-1. Recuperado el 28 de 10 de 2018, de
https://mapserver.sgm.gob.mx/Cartas_Online/geologia/78_E141_GM.pdf

Uribe Alcántara, E.M., Montes León, M.A.L.; García Celis, E., 2010, Mapa Nacional de Îndice de Inundación. Tecnología y Ciencias del Agua, v. 1, n. 2, p. 73-85. ISSN 2007-2422. 